\title{
Anti-PSMA/CD3 BiTE Antibody AMG 160
}

National Cancer Institute

\section{Source}

National Cancer Institute. Anti-PSMA/CD3 BiT E Antibody AMG 160. NCI Thesaurus. Code C157282.

A half-life extended (HLE), bispecific T-cell eng ager (BiTE) antibody composed of two single-chain variable fragments (scFv), one directed against the tumor-associated antigen (TAA) human prostate-specific membrane antigen (PSMA), fused to one that is directed against the CD3 antigen found on T-lymphocytes, with potential immunostimulating and antineoplastic activities. Upon administration of anti-PSMA/CD3 BiTE antibody AMG 160, this bispecific antibody binds to both CD3 on cytotoxic Tlymphocytes (CT Ls) and PSMA found on PSMA-expressing tumor cells. This activates and redirects CT Ls to PSMA-expressing tumor cells, which results in the CT L-mediated cell death of PSMA-expressing tumor cells. PSMA, a tumor associated antigen, is overexpressed on the surface of metastatic and hormone-refractory prostate cancer cells. 